\title{
Von Komma- und anderen Fehlern
}

\author{
Gebräuchliche Medikamente kön- \\ nen zu lebensgefährlichen Kompli- \\ kationen führen. Doch damit alle \\ Beteiligten lernen, die Risiken ein- \\ zuschätzen, müssen die Ursachen \\ offen unter die Lupe genommen \\ werden.
}

W enn wir schon fatale Katastrophen produzieren, sollten wir sie wenigstens publizieren“, forderte Christoph Maier, Bochum. Eines der wenigen Beispiele, die bereits in den 1980er- und 1990erJahren publiziert wurden, war der Fall eines 3,6 kg schweren Säuglings, der ein Multiorganversagen entwickelte, nachdem er innerhalb von 34 Stunden 1,6 g Paracetamol in Form von Zäpfchen erhalten hatte. Der Grund war ein Kommafehler - und unerfahrenes ärztliches wie Pflegepersonal, das den Fehler auch bei mehrmaliger Gabe nicht bemerkte und so fortführte. Immerhin: Das Kind überlebte.

\section{Therapeut als Risikofaktor}

Häufig wird von einem Risikodreieck gesprochen, bestehend aus der Krankheit selbst, dem individuellen Risiko und dem aus den Arzneimitteln erwachsenden. Schonungslos benannte Maier Gründe, warum auch der Schmerztherapeut ein Risikofaktor sein kann: Da wird therapiert, bevor eine Diagnose gesichert ist, Risiken werden bagatellisiert und ein aggressives Helfersyndrom treibt zu vorschnellem Handeln. Und nicht zuletzt wehrte sich Maier gegen die missionarische Selbstwahrnehmung als Helfer gegen den Schmerz als „Volkskrankheit“.

\section{Gene und Metabolisierung}

Ein heikler Aspekt ist die genetisch bedingte unterschiedlich schnelle Verstoffwechselung von Wirkstoffen. So führt die Gabe von Codein als Prodrug von Morphin bei extrem hoher Aktivität von Cytochrom 2D6 bei den „Ultrarapid Metabolizern" zu Nebenwirkungen, während es bei Menschen, die extrem schwach über dieses Enzym verstoffwechseln, überhaupt keine Wirkung zeigt. Daher kann der in gutem Glauben gegebene Codein- haltige Hustensaft bei einem Kind, das ein Ultrarapid Metabolizer ist, sogar zum tödlichen Atemstillstand führen, wie Ulrike Stamer aus Bern, Schweiz, berichtete. Ähnliches kann auch bei der Therapie mit Tramadol passieren, wo bei Ultrarapid Metabolizern die Plasmakonzentration auf das Doppelte der Zieldosis ansteigen kann. Wahrscheinlich ist das unterschiedliche Blutungsrisiko unter nicht steroidalen Antirheumatika ebenfalls abhängig von verschiedenen Cytochrom-Genotypen.

In der Praxis bleibt nur die sorgfältige Kontrolle der Therapie: Wirkt die Subs$\operatorname{tanz}$ und welche Nebenwirkungen treten auf? Keine Wirkung oder unerwartet deutliche Nebenwirkungen können ein Hinweis auf ein genetisches Risiko durch die Metabolisierung sein. Die im Alter häufige Komedikation erhöht über Wechselwirkungen an den Cytochrom-Enzymen das Risiko für unerwünschte Zwischenfälle noch weiter.

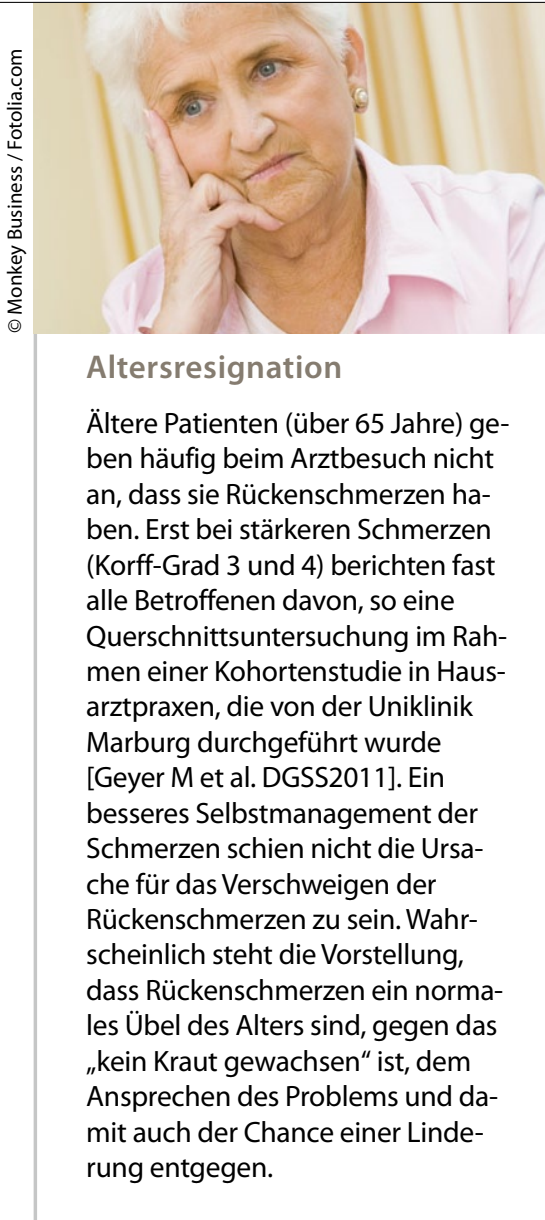

\section{Manchmal muss man sich von} Bewährtem trennen - dies gilt auch für manche in Deutschland häufig eingesetzte Analgetika.

$M$ it deutschen Gewohnheiten räumte Winfried Meißner, Jena, auf: Für das nur in Deutschland zur Therapie akuter Schmerzen verwendet Opiod Piritramid fehle jede Evidenz, betonte er, und auch Codein sei "grottenschlecht wirksam“. Ebenso ist Paracetamol zur Akut-analgesie keine gute Wahl, wie eine aktuelle Cochrane-Metaanalyse, in der die Wirksamkeit verschiedener Analgetika zur Therapie akuter Schmerzen verglichen wurde, belegt: Es müssen vier Patienten mit 1.000 mg Paracetamol behandelt werden, damit einer eine mindestens 50 \%ige Schmerzreduktion über 4-6 h erfährt (Number Needed to Treat $[\mathrm{NNT}]=3,8)$. Bei $120 \mathrm{mg}$ Etoricoxib liegt die NNT dagegen bei nur 1,5. Dabei dauert es bei Etoricoxibgabe (120 mg) we- sentlich länger als bei Paracetamol, bis Patienten die nächste Dosis benötigen (20 vs. 4 h).

\section{Herzgefahr kein Klasseneffekt}

Der seit dem VIOXX-Skandal großen Sorge, Coxibe würden als Klasse insgesamt zu mehr Myokardinfarkten führen, trat Meißner entschieden entgegen. So zeige die retrospektive Auswertung eines dänischen Herzinfarktregisters, dass das Herzinfarktrisiko unter Rofecoxib etwa 2,5-fach erhöht sei, aber auch zum Beispiel bei Diclofenac-Therapie. Deutlich geringer, nämlich nur um $50 \%$, erhöhte sich das Herzinfarktrisiko unter Ibuprofen oder Celecoxib. Meißner warb daher eindringlich dafür, die Herzrisiken der NichtOpiod-Analgetika substanz- und nicht klassenspezifisch zu beurteilen.

Friederike Klein

Berichte vom Deutschen Schmerzkongress

2011 vom 5.-8. Oktober in Mannheim 